\title{
Spectacular Flares of the Radio Galaxy NGC 1275 measured with MAGIC
}

\author{
Dorit Glawion ${ }^{* a}$, Cosimo Nigro ${ }^{b}$, Konstantin Pfrang $^{c}$, Pierre Colin ${ }^{d}$, Vandad Fallah \\ Ramazani $^{e}$, Daniel Mazin ${ }^{f}$, Konstancja Satalecka ${ }^{b}$ for the MAGIC Collaboration \\ ${ }^{a}$ Landessternwarte, Universität Heidelberg \\ Königstuhl, D-69117 Heidelberg, Germany \\ ${ }^{b}$ Deutsches Elektronen-Synchrotron Zeuthen \\ D-15738 Zeuthen, Germany \\ ${ }^{c}$ Technische Universität Dortmund \\ D-44227 Dortmund, Germany \\ ${ }^{d}$ Max-Planck-Institut für Physik \\ D-80805 München, Germany \\ ${ }^{e}$ Finnish MAGIC Consortium, Tuorla Observatory and Finnish Centre for Astronomy with ESO, \\ University of Turku and Astronomy DIvision \\ Univeristy of Oulu, Finland \\ ${ }^{f}$ Japanese MAGIC Consortium \\ Kyoto, Japan \\ E-mail: dglawiondlsw.uni-heidelberg.de, cosimo.nigroddesy.de, \\ konstantin.pfrang@tu-dortmund.de, colin@mppmu.mpg.de, \\ vafara@utu.fi, mazin@icrr.u-tokyo.ac.jp, \\ konstancja.satalecka@desy.de
}

We report on the detection of flaring activity from the prominent Fanaroff-Riley I radio galaxy NGC 1275 located in the Perseus cluster of galaxies in the very-high-energy gamma-ray band. The observations were performed with the MAGIC telescopes between 2016 and 2017 over several months. During this time period, the mean flux above $100 \mathrm{GeV}$ was measured to be roughly ten times brighter than during previous observations. The night-by-night light curve above $100 \mathrm{GeV}$ shows several peaks indicating flux-doubling time-scales of shorter than one day much smaller than the monthly time-scale previously detected by MAGIC. The brightest flux, observed around December 2016 and January 2017, reached 1.75 times the flux from the Crab Nebula, i.e. was about sixty times brighter than the average flux of the previous years. The spectral energy distribution measured up to $>1 \mathrm{TeV}$ shows a curved shape unlike the previously measured simple power-law. The angle between the jet-axis and the line-of sight of NGC 1275 was formerly found to be 30-55 degree in the radio band excluding strong Doppler boosting of the emitted radiation towards the observer. The fast $\mathrm{TeV}$ flux variability and the spectral behaviour observed from a non-blazar object challenge current standard theoretical models and therefore, provide new and fascinating insights into the gamma-ray production and emission mechanism of active galactic nuclei. In the conference, we present the observational results and discuss possible physical processes responsible for the flaring events. 


\section{Misaligned blazars}

The number of extragalactic objects detected in the very-high-energy (VHE, $E>100 \mathrm{GeV}$ ) range with ground-based gamma-ray instruments has grown rapidly in the last twenty years. Out of these, the majority of objects belongs to the class of active galactic nuclei (AGN). As of summer 2017, 70 AGN were found ${ }^{1}$. These objects are radio-loud AGN whose nucleus contains a supermassive black hole and two plasma jets ejected in which particles are believed to be accelerated to relativistic energies and then emit photons through radiative processes. Blazars are AGN with a small angle of $\Theta \sim$ few degrees between the jet axis and the line-of-sight defined by the observer. The majority of the VHE detected AGN belong to the class of blazars. A smaller number of AGN, five in total, is believed to have larger angles $\Theta \gtrsim 10 \mathrm{deg}$. This angle affects the shift of the frequency of the radiation and the boosting of its luminosity. The latter is the reason why AGN with small angles are naturally easier to be detected with current VHE instruments. In case of small angles the flux gets boosted by a few orders of magnitude while for larger angles the flux might be even de-boosted. A strong Doppler boosting due to a small angle and a high bulk Lorentz factor is used to explain the observational findings of blazars such as the fast flux variability $[1,2]$. In this way, one can resolve problems, such as the high opacity due to internal absorption in a tiny emission region, see [3]. However, fast flux variability was also found for the non-blazar objects $[4,5]$. They behave in many aspects like blazars and therefore, those objects are often referred to misaligned blazars.

In this proceeding, we report on the results obtained from MAGIC observations of the object NGC 1275 during the period between September 2016 and February 2017.

\section{NGC 1275}

NGC 1275 is a Fanaroff-Riley (FR) I radio galaxy located in the center of the Perseus cluster of galaxies, better known as 3C 84 in the radio band. The classification as FR I is based on its radio morphology $[6,7]$. Parsec-scale measurements using the Very-Long-Baseline Interferometry technique indicated a viewing angle of $\Theta=30^{\circ}-55^{\circ}$ [8] and $\Theta=65^{\circ} \pm 16^{\circ}$ [9]. On sub-parsec scales one particular radio knot (C3) was found to have been ejected ten years ago and it brightens since then $[10,11]$.

In the optical band, strong nuclear emission lines were found suggesting a Seyfert galaxy $[12,13]$. In [14], NGC 1275 was classified as BL Lac type object, a sub-class of blazars, based on the weak emission-line spectrum, the absence of broad hydrogen lines, and the variability and polarization of the nucleus. Flux variability was found basically in all bands, in radio [15], optical [16], and X-rays [17].

NGC 1275 was first identified to be a gamma-ray emitter with the $\operatorname{COS} B$ satellite [18]. Later on it was detected in the gamma-ray band with Fermi-LAT in the energy range $E>100 \mathrm{MeV}$ [19]. MAGIC and VERITAS reported the detection at $E>100 \mathrm{GeV}$ in [16, 20, 21].

The fastest gamma-ray flux variability was previously found in the LAT data and resulted in time scale of $(1.51 \pm 0.02) \mathrm{d}$ [22], whereas MAGIC measurements only showed marginal flux

\footnotetext{
* Speaker.

${ }^{1}$ http: //tevcat.uchicago.edu/
} 
changes on monthly scales above $100 \mathrm{GeV}$. In October 2016 as well as in January 2017, MAGIC and VERITAS announced the detection of bright flares in the Astronomer's Telegrams \#9689, \#9690, \#9929, and \#9934.

\section{Data analysis and results}

The observations were performed with the MAGIC (Major Atmospheric Gamma Imaging Cherenkov) telescopes. Those are two $17 \mathrm{~m}$-diameter Imaging Atmospheric Cherenkov Telescopes on the island of La Palma, Spain. The energy range covered by standard MAGIC observations is between $50 \mathrm{GeV}$ and $50 \mathrm{TeV}$. The data presented here were gathered under the MAGIC Key Observations Proposal for monitoring misaligned blazars.

During the period September 2016 to February 2017 (MJD 57637.1-57811.9), 56h of observation were performed with the majority of the observations during dark nights and within a zenith distance range of $12^{\circ}<\mathrm{Zd}<50^{\circ}$. For the analysis, we discarded data affected by non-optimal weather conditions and used the standard analysis chain as described in [23].

The analysis of the data resulted in a detection with a significance of $52 \sigma$ calculated with Eq. 17 in [24], see Fig. 1. Here, we show the square route of the distribution of the angular distance between the reconstructed and the expected source position, called $\theta^{2}$, of the signal and background events. We also report for the first time a significant signal from NGC 1275 at energies above $1 \mathrm{TeV}$ with a significance level of $8 \sigma$, see Fig. 2 .

In the time period from September 2016 and February 2017 (MJD 57637.1-57811.9), a mean flux of $(1.19 \pm 0.03) \times 10^{-10} \mathrm{~cm}^{-2} \mathrm{~s}^{-1}$ above $100 \mathrm{GeV}$ was measured with the MAGIC telescopes. This is seven to nine times higher than during previous observations in 2009-2010 and 2010-2011 reported [16] where a typical flux of $\sim 3 \%$ of the Crab Nebula flux was measured. We found a high state in October-November 2016 as reported in the ATel \#9689 and again strong flaring activity in December 2016 and January 2017 with one exceptional outburst announced in ATel \#9929 with a flux of 1.75 times the Crab Nebula flux above $100 \mathrm{GeV}$. The latter outburst was 60 times brighter than the low state measured in [16].

\section{Discussion}

The so-called shock-in-jet model is used to explain the production of gamma-rays in AGN. In this model, particles are accelerated to high energies via shock acceleration at shock fronts in the jets of the AGN. The observed radiation is believed to be produced by, e.g., synchrotron-self Compton (SSC) processes [25, 26, 27]. The simplest version of such a model assumes a single spherical component in which synchrotron photons are producing the low energy emission and the same low energy photons are seeds for the inverse-Compton emission observed in the high-energy bands. In case of fast flux variability, the size of the emission region becomes small, and VHE emission can be absorbed via internal $\gamma \gamma$-pair production. Strong Doppler boosting can solve this problem [3].

In case of NGC 1275, we have investigated the variability time scales and the corresponding Doppler factor necessary to maintain the observed emission. We found that rather moderate 


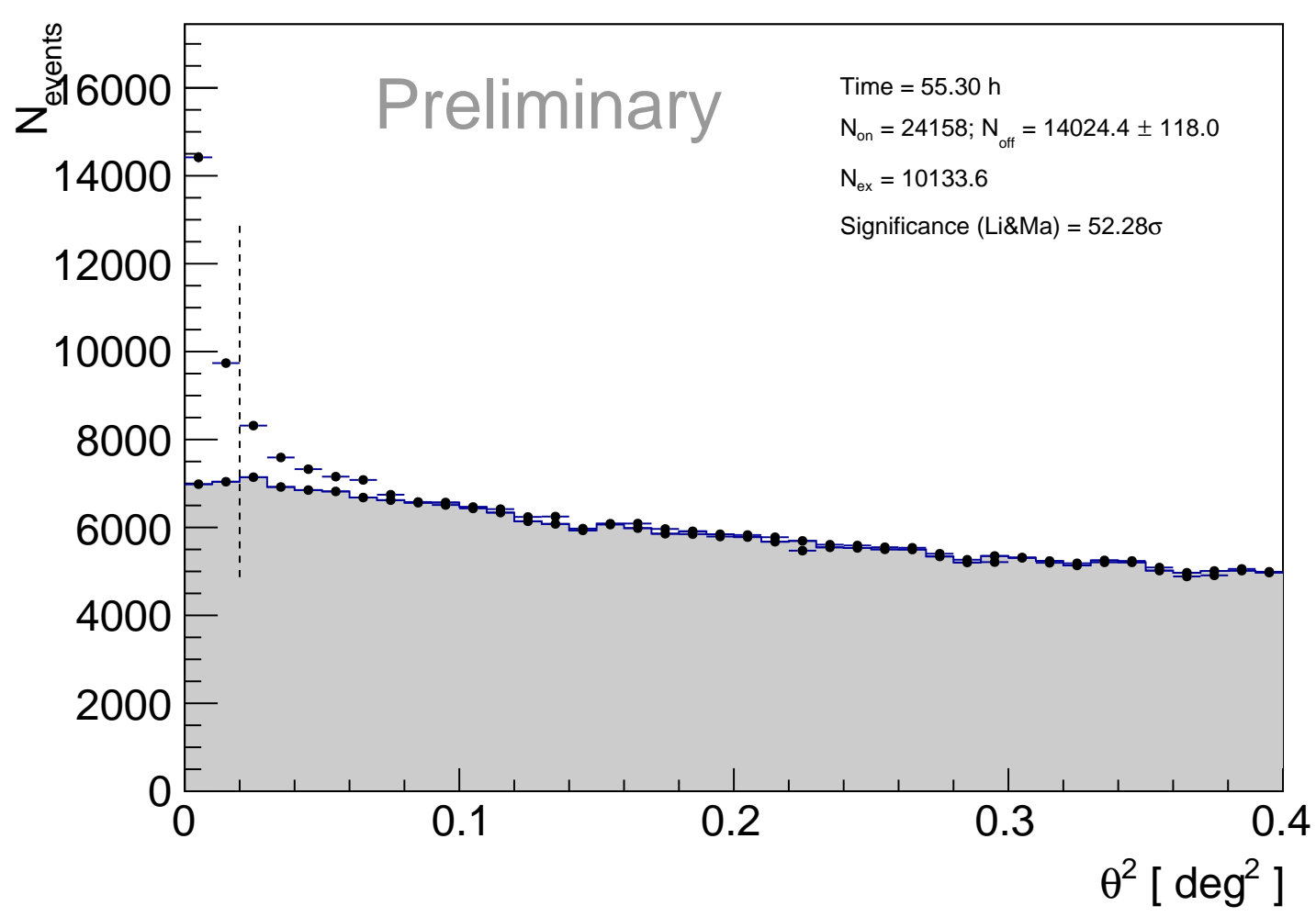

Figure 1: $\theta^{2}$ distributions of the signal (black points) and background (gray area) estimation performed between September 2016 and February 2017. The significance is calculated for the region between zero and the vertical dashed line.

Doppler factors $\delta \sim \mathscr{O}\left(10^{0-1}\right)$ are needed which however constrain the viewing angle to be much smaller than the angles yielded in radio observations.

In [28] a spine-layer geometry of the emission zone was used to explain the broad-band radiation of NGC 1275. In this model, a fast spine with $\Gamma_{\text {spine }}=10-20$ in the inner part of the cylindrical jet is surrounded by a slower layer with $\Gamma_{\text {layer }}=2-4$. The VHE emission is produced predominantly by the latter. A limb-brightened structure of the inner parsec-scale jet of NGC 1275 was reported in [29] which indicates such a geometry.

The authors mentioned that $\mathrm{TeV}$ emission may not be explained by their model due to strong internal pair-production. As we clearly found a signal at energies greater than $1 \mathrm{TeV}$, such an model can probably be excluded as explanation for the flaring activity.

Alternative scenarios [30] such as the magnetospheric models [31, 32], star-in-jet interactions $[33,34,35]$ or magnetic reconnection $[36,37]$ would be investigated in the future.

\section{Conclusion}

More and more gamma-ray observations of misaligned blazars (e.g., fast variability and hard $\mathrm{TeV}$ spectra) are reported which are not easy to be explained with our standard models for the gamma-ray production in AGN and therefore, alternative models needs to be identified or exploit to 


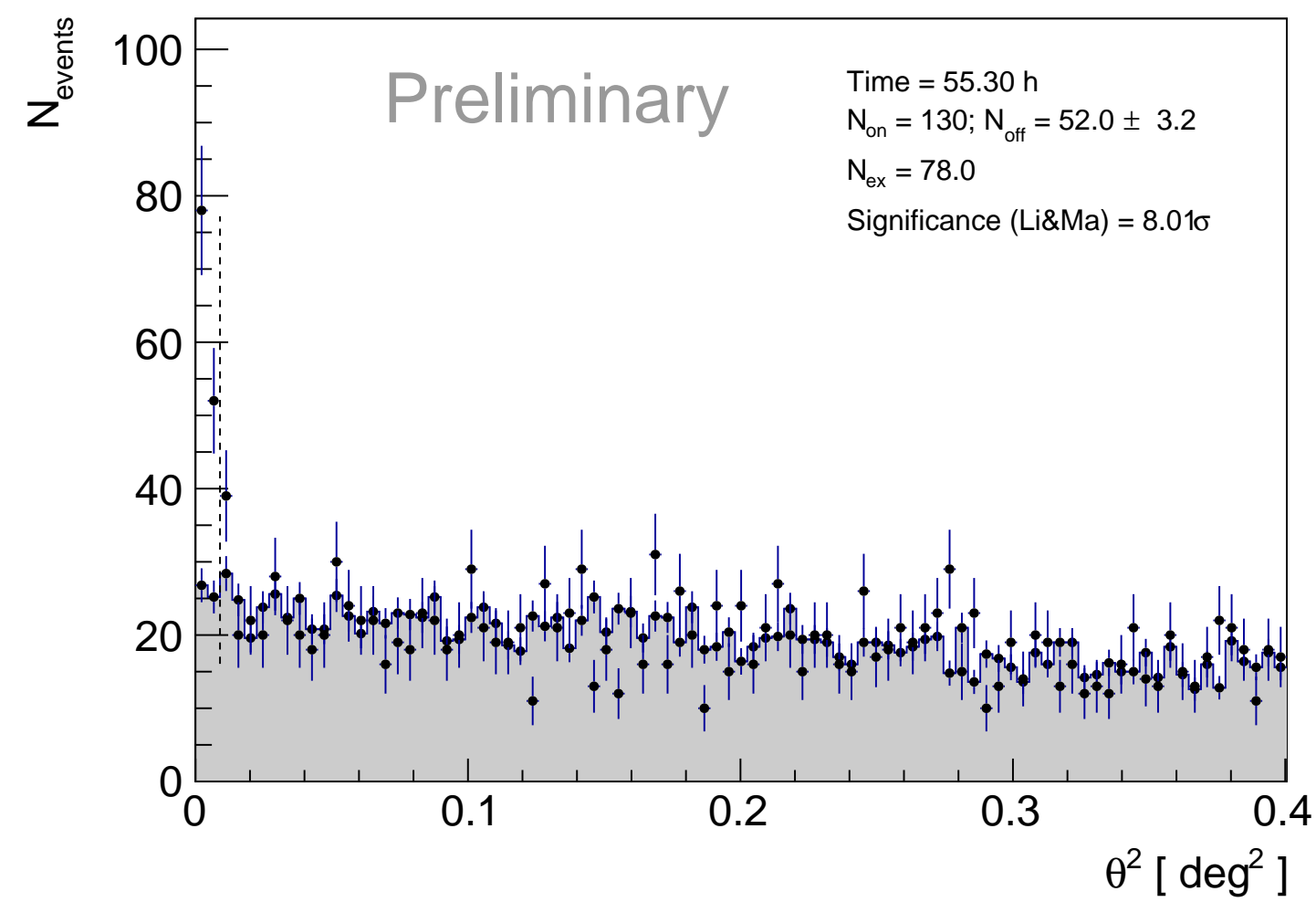

Figure 2: $\theta^{2}$ distributions of the signal (black points) and background (gray area) estimation above an energy of $1 \mathrm{TeV}$ from observations performed between September 2016 and February 2017. The significance is calculated for the region between zero and the vertical dashed line.

deepen our understanding of the production of gamma-ray emission in AGN. Studying this requires a preferably continuous monitoring with Fermi-LAT and the ground-based gamma-ray instruments. This, together with multi-wavelength observations as well as deep VLBI measurements will enlarge our knowledge of the jet structure and the connection between the jet and central black hole and their surroundings.

\section{Acknowledgments}

We would like to thank the Instituto de Astrofísica de Canarias for the excellent working conditions at the Observatorio del Roque de los Muchachos in La Palma. The financial support of the German BMBF and MPG, the Italian INFN and INAF, the Swiss National Fund SNF, the ERDF under the Spanish MINECO (FPA2015-69818-P, FPA2012-36668, FPA2015-68378-P, FPA2015-69210-C6-2-R, FPA2015-69210-C6-4R, FPA2015-69210-C6-6-R, AYA2015-71042-P, AYA2016-76012-C3-1-P, ESP2015-71662-C2-2-P, CSD200900064), and the Japanese JSPS and MEXT is gratefully acknowledged. This work was also supported by the Spanish Centro de Excelencia "Severo Ochoa" SEV-2012-0234 and SEV-2015-0548, and Unidad de Excelencia "Maria de Maeztu" MDM-2014-0369, by the Croatian Science Foundation (HrZZ) Project 09/176 and the University of Rijeka Project 13.12.1.3.02, by the DFG Collaborative Research Centers SFB823/C4 and SFB876/C3, and by the Polish MNiSzW grant 2016/22/M/ST9/00382.

D.G. acknowledges the financial support of the German Academic Exchange Service (DAAD) and from German BMBF (Fkz. 5A14VH4). 


\section{References}

[1] Aharonian, F., Akhperjanian, A. G., Bazer-Bachi, A. R. et al. An Exceptional Very High Energy Gamma-Ray Flare of PKS 2155-304, ApJ 664 (2007) L71

[2] Albert, J., Aliu, E., Anderhub, H., et al. Variable Very High Energy $\gamma$-Ray Emission from Markarian 501, ApJ 669 (2007) 862

[3] Begelman, M. C., Fabian, A. C., \& Rees, M. J. Implications of very rapid TeV variability in blazars, MNRAS 384 (2008) L19

[4] Acciari, V. A., Aliu, E., Arlen, T., et al. Radio Imaging of the Very-High-Energy $\gamma$-Ray Emission Region in the Central Engine of a Radio Galaxy, Science 325 (2009) 444

[5] Aleksić, J., Ansoldi, S., Antonelli, L. A., et al. Black hole lightning due to particle acceleration at subhorizon scales, Science 346 (2014c) 1080

[6] Vermeulen, R. C., Readhead, A. C. S., \& Backer, D. C. Discovery of a nuclear counterjet in NGC 1275: A new way to probe the parsec-scale environment, ApJ 430 (1994) L41

[7] Buttiglione, S., Capetti, A., Celotti, A., et al. An optical spectroscopic survey of the 3CR sample of radio galaxies with $z<0.3$. II. Spectroscopic classes and accretion modes in radio-loud AGN, A\&A 509 (2010) A6

[8] Walker, R. C., Romney, J. D., \& Benson, J. M. Detection of a VLBI counterjet in NGC 1275: A possible probe of the parsec-scale accretion region, ApJ 430 (1994) L45

[9] Fujita, Y. \& Nagai, H. Discovery of a new subparsec counterjet in NGC 1275: the inclination angle and the environment, MNRAS 465 (2017) L94

[10] Nagai, H., Suzuki, K., Asada, K., et al. VLBI Monitoring of $3 C 84$ (NGC 1275) in Early Phase of the 2005 Outburst, PASJ 62 (2010) L11

[11] Nagai, H., Orienti, M., Kino, M., et al. VLBI and single-dish monitoring of $3 C 84$ for the period 2009-2011, MNRAS 423 (2012) L122

[12] Humason, M. L. The Emission Spectrum of the Extra-Galactic Nebula N.G.C. 1275, PASP 44 (1932) 267

[13] Khachikian, E. Y. \& Weedman, D. W. An atlas of Seyfert galaxies, ApJ 192 (1974) 581

[14] Veron, P. NGC1275 - A BL Lacertae object?, Nature 272 (1978) 430

[15] Dutson, K. L., Edge, A. C., Hinton, J. A., et al. A non-thermal study of the brightest cluster galaxy NGC 1275 - the Gamma-Radio connection over four decades, MNRAS 442 (2014) 2048

[16] Aleksić, J., Ansoldi, S., Antonelli, L. A., et al. Contemporaneous observations of the radio galaxy NGC 1275 from radio to very high energy $\gamma$-rays, A\&A 564 (2014) A5

[17] Fukazawa, Y., Shiki, K., Tanaka, Y., Itoh, R., \& Nagai, H. X-ray variability of GeV gamma-ray emitting radio galaxy NGC 12752016 ArXiv e-prints [arXiv:1608.03652]

[18] Strong, A. W., Bignami, G. F., Caraveo, P. A., et al. The local interstellar medium as traced by gamma rays, $A \& A 115$ (1982) 404

[19] Abdo, A. A., Ackermann, M., Ajello, M., et al. Fermi Discovery of Gamma-ray Emission from NGC 1275, ApJ 699 (2009) 31

[20] Aleksić, J., Alvarez, E. A., Antonelli, L. A., et al. Detection of very-high energy $\gamma$-ray emission from NGC 1275 by the MAGIC telescopes, A\&A 539 (2012a) L2 
[21] Benbow, W. for the VERITAS Collaboration. Highlights from the VERITAS AGN Observation Program 2015 ArXiv e-prints [arXiv:1508.07251]

[22] Brown, A. M. \& Adams, J. High-energy $\gamma$-ray properties of the Fanaroff-Riley type I radio galaxy NGC 1275, MNRAS 413 (2011) 2785

[23] Aleksić, J., Ansoldi, S., Antonelli, L. A., et al. The major upgrade of the MAGIC telescopes, Part II: A performance study using observations of the Crab Nebula, Astroparticle Physics 72 (2016) 76

[24] Li, T.-P. \& Ma, Y.-Q. Analysis methods for results in gamma-ray astronomy, ApJ 272 (1983) 317

[25] Marscher, A. P. \& Gear, W. K. Models for high-frequency radio outbursts in extragalactic sources, with application to the early 1983 millimeter-to-infrared flare of 3C 273, ApJ 298 (1985) 114

[26] Maraschi, L. \& Ghisellini, G. \& Celotti, A. A jet model for the gamma-ray emitting blazar 3C 279, ApJL 397 (1992) L5

[27] Bloom, S. D. \& Marscher, A. P. An Analysis of the Synchrotron Self-Compton Model for the Multi-Wave Band Spectra of Blazars, ApJ 461 (1996) 657

[28] Tavecchio, F. \& Ghisellini, G. On the spine-layer scenario for the very high-energy emission of NGC 1275, MNRAS 443 (2014) 1224

[29] Nagai, H., Haga, T., Giovannini, G., et al. Limb-brightened Jet of $3 C 84$ Revealed by the $43 \mathrm{GHz}$ Very-Long-Baseline-Array Observation, ApJ 785 (2014) 53

[30] Aharonian, F. A., Barkov, M. V., \& Khangulyan, D. Scenarios for Ultrafast Gamma-Ray Variability in $A G N$, ApJ 841 (2017) 61

[31] Levinson, A. \& Rieger, F. Variable TeV Emission as a Manifestation of Jet Formation in M87?, ApJ 730 (2011) 123

[32] Hirotani, K. \& Pu, H.-Y. Energetic Gamma Radiation from Rapidly Rotating Black Holes, ApJ 818 (2015) 50

[33] Bednarek, W. \& Protheroe, R. J. Gamma-rays from interactions of stars with active galactic nucleus jets, MNRAS 287 (1997) L9

[34] Barkov, M. V., Aharonian, F. A., Bogovalov, S. V., et al. Interpretation of the Flares of M87 at TeV Energies in the Cloud-Jet Interaction Scenario, ApJ 749 (2012a) 119

[35] Barkov, M. V., Bosch-Ramon, V., \& Aharonian, F. A. Rapid TeV Variability in Blazars as a Result of Jet-Star Interaction, ApJ 755 (2012b) 170

[36] Giannios, D., Uzdensky, D. A., \& Begelman, M. C. Fast TeV variability in blazars: jets in a jet, MNRAS 395 (2009) L29

[37] Giannios, D., Uzdensky, D. A., \& Begelman, M. C. Fast TeV variability from misaligned minijets in the jet of M87, MNRAS 402 (2010) 1649 